\title{
Detection of Alcohol Vapor Using Surface Plasmon Resonance Sensor with Organic-Inorganic Hybrid Layers
}

\author{
Tsuyoshi Arakawa*, Hisao Yasukawa and Katsuya Fujimoto \\ Department of Biological and Environmental Chemistry, \\ Faculty of Humanity-Oriented Science and Engineering, Kinki University, \\ 11-6 Kayanomori, Iizuka, Fukuoka 820-8555, Japan
}

(Received September 29, 2009; accepted April 21, 2010)

Key words: Zirconium phosphate, titanium oxide, 2 aminoethanethiol, (3-mercaptopropyl) trimethoxysilane, alcohol vapor, surface plasmon resonance sensor

The detection of alcohol vapor using a surface plasmon resonance (SPR) sensor with an organic-inorganic hybrid was investigated. An alkyl thiol thin film alone had no sensitivity to alcohol vapor. However, when zirconium phosphate via hydroxyl aminoethanethiol interfacial layers or $\mathrm{TiO}_{2}$ via silanethiol layers was formed, a large change in incident angle was observed. The incident angle caused by the adsorption of alcohol vapor $(\Delta \theta)$ increased with an increase in the concentration of alcohol vapor. The sequence of the sensitivity was as follows: 2-butanol $>1$-propanol $>$ ethanol $>$ methanol.

\section{Introduction}

Surface plasmon resonance (SPR) is one of the most promising optical techniques for chemical sensing. The surface plasmon has been studied both theoretically and experimentally..$^{(1,2)}$ Since resonance angle is very sensitive to the outside of a metal thin film, the refractive index of polymeric films, organic compounds, and inorganic compounds can be obtained by SPR..$^{(3,4)}$ In particular, a substrate coated with a gold thin film of the SPR sensor head affects the sensitivity or selectivity for the detection of gaseous species. Nylander et al. ${ }^{(5)}$ reported a unique example of gas detection using a thin oil film of a silicon-glycol copolymer with a high solubility for halogenated hydrocarbons. In addition, it was reported that a $\mathrm{TiO}_{2}$ nanocrystal thin film deposited onto Au/glass as the sensing layer exhibited a highly sensitive and reproducible SPR response to alcohol vapor. ${ }^{(6)}$

Recently, traffic accidents due to drunk driving have become an increasingly serious social problem. One measure to solve this problem is the use of an "alcohol checker" before driving. Alcohol checkers using semiconductor methods are already sold by many manufacturers. We have attempted to detect certain gaseous species using an ${ }^{*}$ Corresponding author: e-mail: arakawa@fuk.kindai.ac.jp 
SPR sensor and proposed a new method for the detection of alcohol vapor. When a polyethylene glycohol layer was coated on a Au thin film, it was found that alcohol could be selectively detected.(7) In addition, organic-inorganic hybrids as sensor materials have received considerable attention ${ }^{(8,9)}$ because organic-inorganic hybridization is considered an effective method for improving sensitivity or selectivity. When a zeolite thin film on a gold surface via an anthiol-alkoxysilane interfacial layer was formed, it was found that amines could be selectively detected. ${ }^{(10)}$ In this paper, we describe the detection of alcohol vapor using some inorganic-organic hybrids. We selected zirconium phosphate $\mathrm{HZr}_{2}\left(\mathrm{PO}_{4}\right)_{3}$ or $\mathrm{TiO}_{2}$ as the inorganic material, because these compounds are expected to exhibit typical surface properties suitable for alcohol vapor detection.

\section{Experimental Procedure}

Figure 1 shows the block diagram of the apparatus used for an SPR-based chemical sensor to be identical to that reported elsewhere. ${ }^{(11)}$ The incident angle of the He-Ne laser beam at the metal from the prism is $\theta$. The beam reflected at the sensor face of the prism, is again reflected at the other surface of the prism, which is mirror-coated, and returns back to the photodiode detector. The prism with the gas sample cell is rotated about a point of the sensing part of the metal film. The sensing head is rotated by a stepping motor to change the incident angle $\theta$ of the He-Ne laser beam. The measurement sequence is controlled by a personal computer. The sensing head was rotated by a stepping motor with a resolution of $0.05^{\circ}$. A sensor head was prepared as follows. A gold thin film with a thickness of about $55 \mathrm{~nm}$ was prepared by the thermal evaporation of gold (purity, 99.99\%) on the surface of a corner cube prism. The anchoring of commercially available zirconium phosphate $\mathrm{HZr}_{2}\left(\mathrm{PO}_{4}\right)_{3}$ crystals to the gold surface involved two steps: formation of thiol monolayer and exposure to a zirconium phosphate

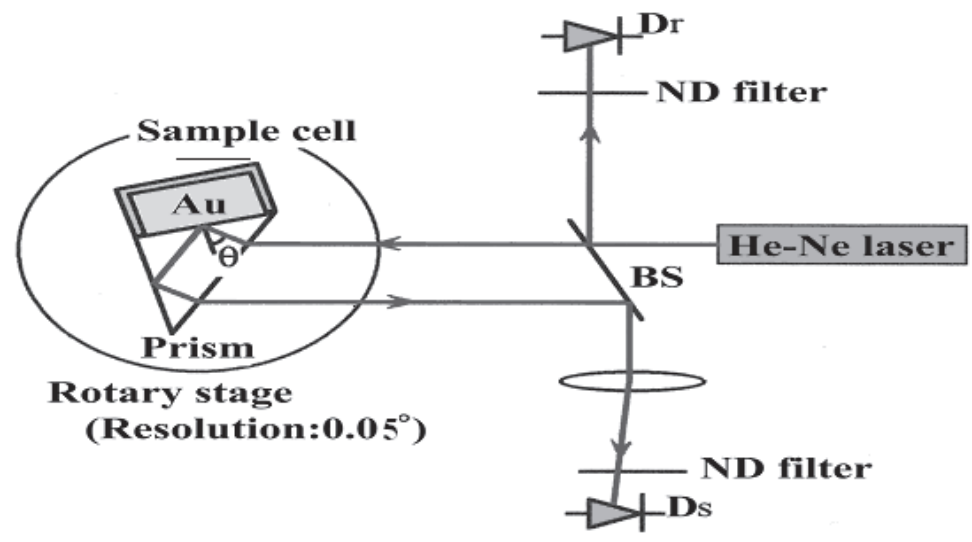

Fig. 1. Block diagram of the optical part of a surface-plasmon-resonance-based chemical sensor. 
crystals suspension in toluene, followed by the attachment of a gas sample cell. $\mathrm{HZr}_{2}\left(\mathrm{PO}_{4}\right)_{3}$, 2-aminoethanethiol, starch, and sodium borohydride were purchased from Wako Pure Chemical Industries Ltd. A prism coated with a gold thin film was immersed in 2-aminoethanethiol ethanol solution for $24 \mathrm{~h}$ to form a self-assembled monolayer via Au-mercaptide bonding. Then, it was treated with a mixed solution of soluble starch and periodic acid, followed by immersion in sodium borohydride solution in order to form a surface hydroxyl group in a manner similar to that reported elsewhere. ${ }^{(12)}$ The thiol layer was rinsed with toluene to remove excess adsorbate from the gold thin film, followed by stirring in $30 \mathrm{ml}$ of toluene solution of dispersed zirconium phosphate crystals $(0.1 \mathrm{~g})$ overnight. After attachment of the zirconium phosphate crystals, the $\mathrm{OH}$ group of the alkyl thiol was reacted with zirconium phosphate by heating in air at $373 \mathrm{~K}$ for $1 \mathrm{~h}$.

Anatase $\mathrm{TiO}_{2}$ was synthesized by the hydrolysis of $\left(\mathrm{NH}_{4}\right)_{2} \mathrm{TiF}_{6}$ (Sigma-Aldrich Co.) as shown in the following reactions:

$$
\begin{gathered}
\left(\mathrm{NH}_{4}\right)_{2} \mathrm{TiF}_{6}+2 \mathrm{H}_{2} \mathrm{O} \rightarrow \mathrm{TiO}_{2}+4 \mathrm{HF}+\left(\mathrm{NH}_{4}\right)_{2} \mathrm{~F}_{2} \\
\mathrm{~B}_{2} \mathrm{O}_{3}+3 \mathrm{H}_{2} \mathrm{O} \rightarrow 2 \mathrm{H}_{3} \mathrm{BO}_{3} \\
\mathrm{H}_{3} \mathrm{BO}_{3}+4 \mathrm{HF} \rightarrow \mathrm{BF}_{4}^{-}+\mathrm{H}_{3} \mathrm{O}++2 \mathrm{H}_{2} \mathrm{O} .
\end{gathered}
$$

When $0.07 \mathrm{M} \mathrm{B}_{2} \mathrm{O}_{3}$ was added to $0.01 \mathrm{M}$ aqueous solution of $\left(\mathrm{NH}_{4}\right)_{2} \mathrm{TiF}_{6}, \mathrm{TiO}_{2}$ was deposited, followed by the separation using a centrifuge and drying of the precipitate formed in a desiccator. The anchoring of $\mathrm{TiO}_{2}$ crystals to the gold surface involved two steps: formation of the thiol layers and exposure to a $\mathrm{TiO}_{2}$ suspension in toluene. The thiol layers were formed by immersing the gold thin film in a solution containing $0.1 \mathrm{~g}$ of (3-mercaptopropyl) trimethoxysilane (Wako Pure Chemical Industries Ltd.) dissolved in $30 \mathrm{ml}$ of toluene for $1 \mathrm{~h}$. Then, the thiol layers were rinsed with toluene to remove excess adsorbate from the gold thin film, followed by stirring in $90 \mathrm{ml}$ of toluene of dispersed $\mathrm{TiO}_{2}(0.3 \mathrm{~g})$ overnight. After the attachment of the gas sample cell, a fixed quantity of alcohol vapor mixed with dry air at various concentrations $(0.1-0.5 \%)$ was introduced from one side of the gas sample cell with a gas syringe. SEM images were obtained using a Hitachi S-4300SE/N.

\section{Results and Discussion}

\subsection{Formation of zirconium phosphate crystal}

The zirconium phosphate $\mathrm{HZr}_{2}\left(\mathrm{PO}_{4}\right)_{3}$ has a three-dimensional skeleton structure composed of octahedral $\mathrm{ZrO}_{4}$ and tetrahedral $\mathrm{PO}_{4} \cdot{ }^{(13)}$ Fig. 2(a) presents an SEM image of the zirconium-phosphate-crystal-coated gold surface on a glass slide instead of a prism. It is clear that this coating process provides a method of obtaining uniform crystal distributions on the entire glass slide surface. Small crystals $(\sim 0.5 \mu \mathrm{m})$ condensed to form rodlike masses, and there were small gaps between rodlike masses. In addition, in the case of a $\mathrm{TiO}_{2}$-organic hybrid, small $\mathrm{TiO}_{2}$ particles $(\sim 0.3 \mu \mathrm{m})$ assembled to form rodlike masses similar to those of a $\mathrm{HZr}_{2}\left(\mathrm{PO}_{4}\right)_{3}$ hybrid (Fig. 2(b)). It was independently confirmed that the zirconium phosphate crystals or anatase $\mathrm{TiO}_{2}$ recovered from the gold substrate gave the same X-ray diffraction pattern peak ratios as the bulk. Although the 


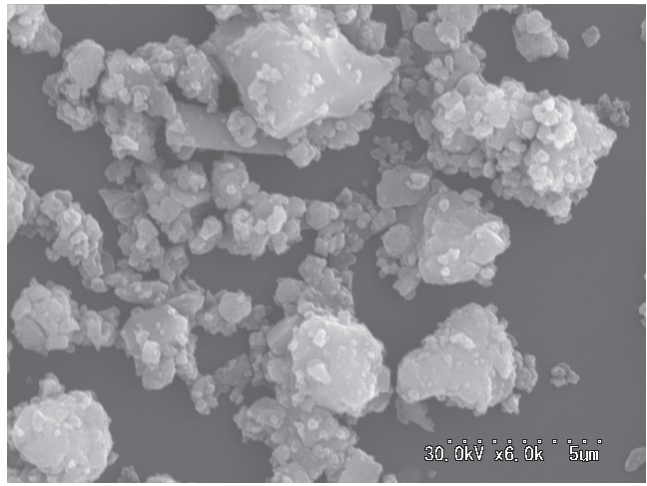

(a)

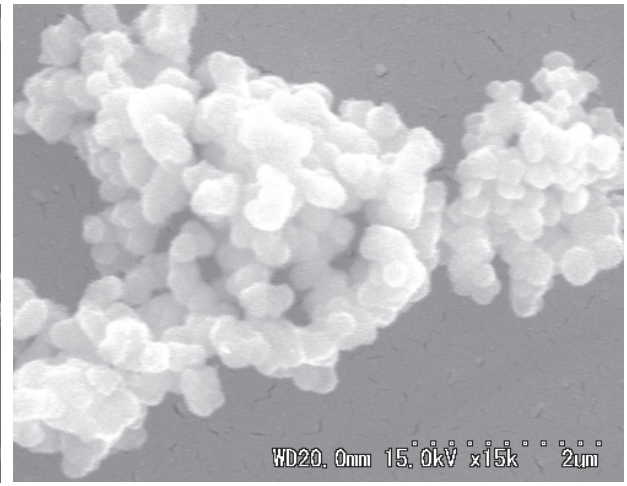

(b)

Fig. 2. (a) SEM image of zirconium phosphate crystals on gold surface via the interfacial coupling layer. (b) SEM image of $\mathrm{TiO}_{2}$ crystals on gold surface via the interfacial coupling layer.

structure of the hybrid are not well-defined, we propose the structure of the hybrid as shown in Figs. 3(a) and 3(b). The dehydration reaction proceeds between the $\mathrm{OH}$ group of aminoethanethiol and the $\mathrm{H}^{+}$of $\mathrm{HZr}_{2}\left(\mathrm{PO}_{4}\right)_{3}$. On the other hand, the $\mathrm{OH}$ group of the $\mathrm{TiO}_{2}$ surface as described below reacted with the $-\mathrm{OCH}_{3}$ group of (3-mercaptopropyl) trimethoxysilane. Although the number of $\mathrm{HZr}_{2}\left(\mathrm{PO}_{4}\right)_{3}$ or $\mathrm{TiO}_{2}$ molecules in the inorganic layer is not proved, the surface state of these hybrid layers differs from one another in chemical property as shown in the figures.

\subsection{SPR spectra under adsorption of alcohols}

In SPR, resonance angle is very sensitive to the refractive index of the medium outside the metal thin film. In addition, the resonance angle of the Au/prism varies with the refractive index of the prism, as shown in Figs. 4 and 5. When the medium on the surface of the gold thin film was changed from air to a 2-aminoethanethiol thin film, resonance angle changed from $24.2^{\circ}$ to $25.5^{\circ}$ for $n=1.880$. On the other hand, when the gold surface was coated with (3-mercaptopropyl) trimethoxysilane, resonance angle varied from $18.80^{\circ}$ to $22.90^{\circ}$ for $n=1.883$. In the process by which the tail group of thiol was changed from $-\mathrm{NH}_{2}$ to $-\mathrm{CH}_{2} \mathrm{OH}$, the peak of the resonance curve became $27.1^{\circ}$. Moreover, after the formation of a thiol-zirconium phosphate hybrid layer or a $\mathrm{TiO}_{2}$ hybrid layer, the peak of the resonance curve shifted to a larger incident angle, as shown in Figs. 4 and 5. Next, for the measurement of ethanol vapor with a $\mathrm{TiO}_{2}$ hybrid, the change in $\Delta \theta$ after admitting ethanol vapor ( 0.05 and $0.1 \mathrm{vol} \%)$ and displacing air is shown in Fig. 6. When the alcohol vapor was introduced into the gas sample cell, incident angle immediately shifted and continued to slowly shift to a higher value for about $15 \mathrm{~min}$. Incident angle increased from methanol to 2-butanol. Moreover, when the atmosphere was changed from alcohol vapor back to air, incident angle decreased with time and returned to its original value after about $15 \mathrm{~min}$. After many repeated 
(a)

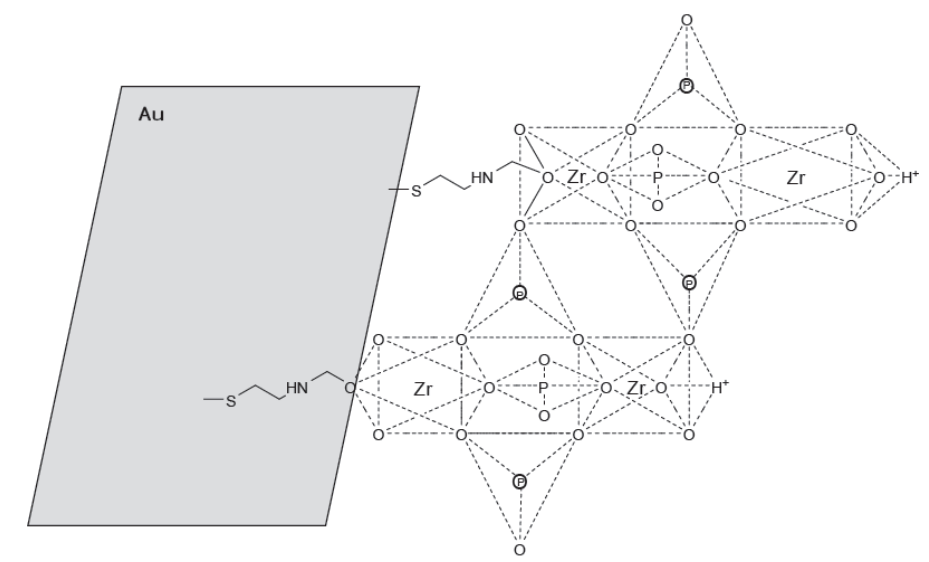

(b)

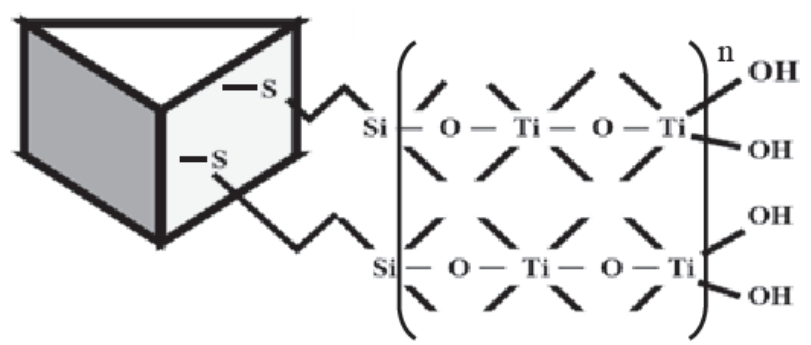

Fig. 3. (a) Schematic diagrams of $\mathrm{HZr}_{2}\left(\mathrm{PO}_{4}\right)_{3}$ hybrid layer and (b) $\mathrm{TiO}_{2}$ hybrid layer on $\mathrm{Au}$ thin film.

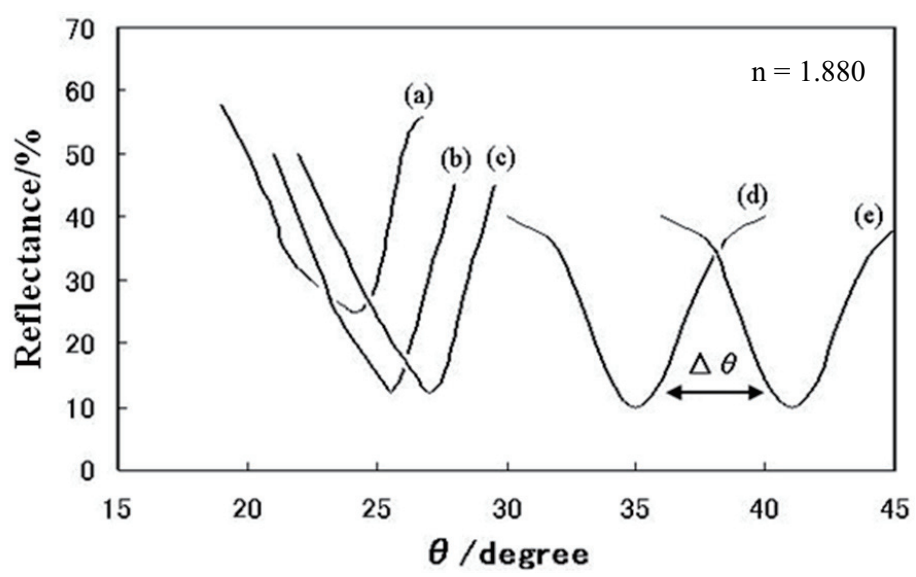

Fig. 4. Resonance curves for a gold film with a prism $(n=1.880)$ : (a) in air $\left(24.2^{\circ}\right)$, (b) coated with the thiol film with $-\mathrm{NH}_{2}$ as the tail group $\left(25.5^{\circ}\right)$, (c) coated with the thiol film with $-\mathrm{CH}_{2} \mathrm{OH}$ as the tail group $\left(27.1^{\circ}\right)$, (d) coated with the chemically anchored zirconium phosphate layer $\left(35.0^{\circ}\right)$, and (e) after introducing methanol vapor $\left(\sim 2 \%, 41.0^{\circ}\right)$. 


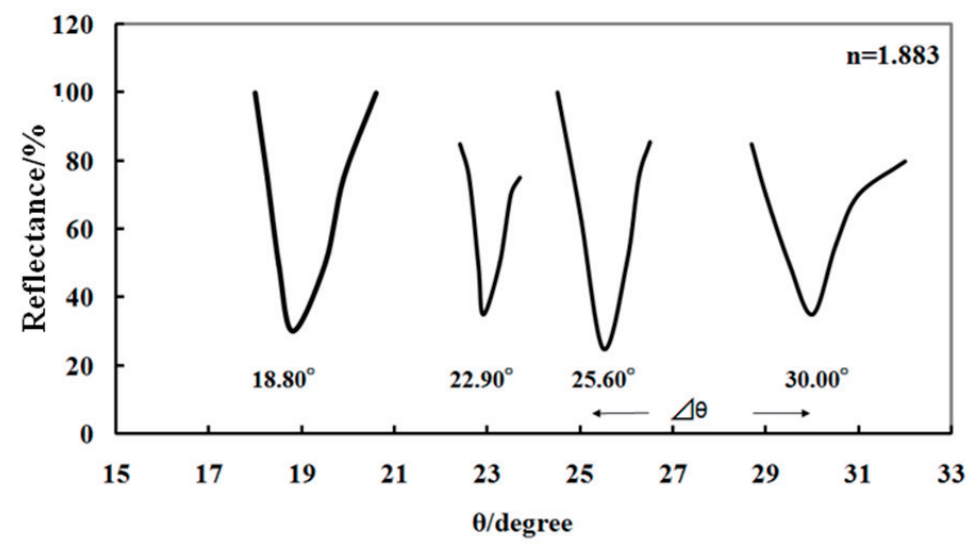

Fig. 5. Resonance curves for a gold film with a prism $(n=1.883)$ : (a) in air $\left(18.80^{\circ}\right)$, (b) coated with the (3-mercaptopropyl) trimethoysilane $\left(22.90^{\circ}\right)$, (c) coated with the chemically anchored $\mathrm{TiO}_{2}$ layer $\left(25.60^{\circ}\right)$, and (d) after introducing methanol vapor $\left(0.1 \%, 30.00^{\circ}\right)$.

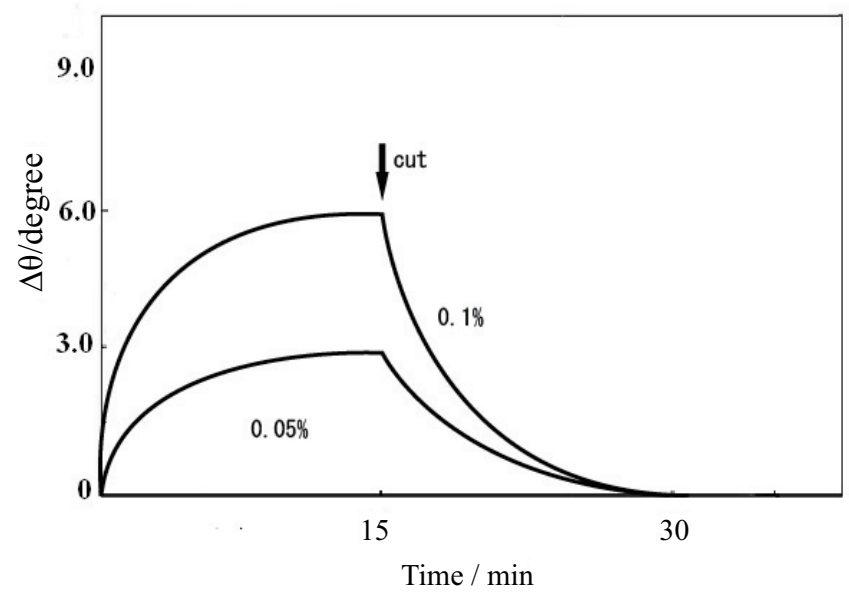

Fig. 6. Time response of the incident angle shift upon exposure of the $\mathrm{TiO}_{2}$ hybrid layer to ethanol vapor $(0.05$ and $0.1 \mathrm{vol} \%)$.

experiments, the reproducibility of incident angle was confirmed. For example, the relative standard deviation for 5 measurements of $0.1 \mathrm{vol} \% \mathrm{C}_{2} \mathrm{H}_{5} \mathrm{OH}$ was $2.1 \%$. In the case of only the alkyl thiol thin film with $-\mathrm{CH}_{2} \mathrm{OH}$ as the tail group or (3-mercaptopropyl) trimethoxysilane thin film, a shift in incident angle after the introduction of alcohol vapor was hardly observed. Thus, the change in the incident angle is attributed to the adsorption or desorption of alcohol on the zirconium phosphate or $\mathrm{TiO}_{2}$ layer. 
The shift in incident angle after gas adsorption $(\Delta \theta)$ increased with the concentration of alcohol vapor, as shown in Figs. 7 and 8. In the case of the zirconium phosphate hybrid layer, $\Delta \theta$ increased linearly with the concentration of alcohol vapor in the investigated range. However, for the SPR sensor with the $\mathrm{TiO}_{2}$ hybrid layer, $\Delta \theta$ increased linearly up to $0.1 \%$ and beyond $0.1 \%$ was slightly changed, except for 2-butanol. The slope of the straight line for $\mathrm{C}_{2} \mathrm{H}_{5} \mathrm{OH}$ with the $\mathrm{TiO}_{2}$ hybrid is about 200 $\mathrm{ppm} / \mathrm{deg}$. Thus, we can estimate the lower limit of detection for our simple equipment

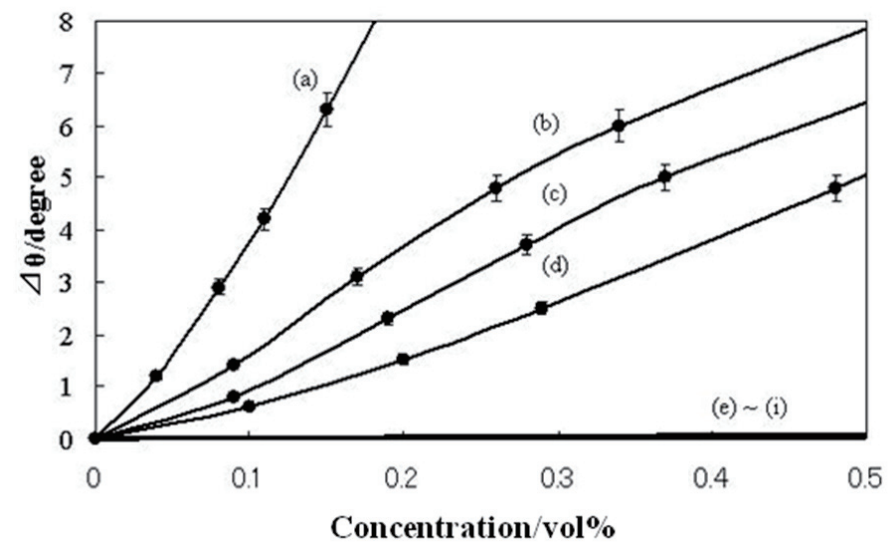

Fig. 7. Calibration plots of the alcohols, ketones and aldehydes: (a) 2-butanol (17.2), (b) 1-propanol (20.3), (c) ethanol (24.6), (d) methanol (32.6), (e) acetone, (f) ethyl methyl ketone, (g) butanal, (i) propionaldehyde, and (j) acetaldehyde. The values in parentheses are the dielectric constants.

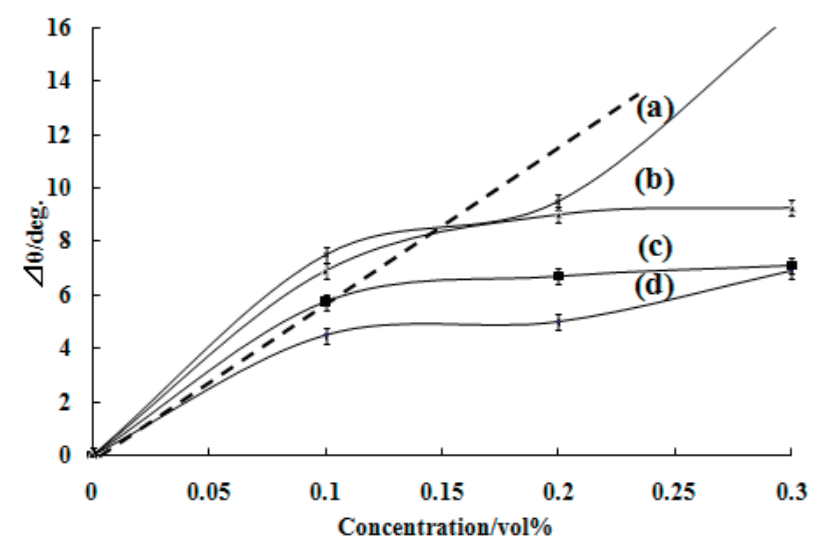

Fig. 8. Calibration plots of the alcohols: (a) 2-butanol, (b) 1-propanol, (c) ethanol, and (d) methanol. 
to be approximately $4 \mathrm{ppm}$, judging from the resolution of the rotary stage. The slope of the straight line and the lower limit of detection for $\mathrm{C}_{2} \mathrm{H}_{5} \mathrm{OH}$ with zirconium phosphate hybrid are about $1000 \mathrm{ppm} /$ degree and ca. $20 \mathrm{ppm}$, respectively. These values are comparable to the limited data achieved using the commercial alcohol checker. The slope of the straight line refers to the sensitivity. The slope depends on the species of alcohol, and the sensitivity of 2-butanol was highest among certain alcohols in both cases; the sequence of sensitivity was as follows: 2-butanol (17.2) > 1-propanol (20.3) $>$ ethanol (24.6) > methanol (32.6). The values in parentheses are dielectric constants. The shift increases with increasing carbon number and the dielectric constant decreases with increasing carbon number of alcohol. The sequence of sensitivity is similar to that of polyethylene glycol $(n=600)$ thin film as reported elsewhere. ${ }^{(6)}$ Although there was a difference in sensitivity between the zirconium phosphate hybrid and the $\mathrm{TiO}_{2}$ hybrid, the sensitivity of these hybrids was higher than that of polyethylene glycol $(n=600)$ thin film.

Since there are $\mathrm{H}^{+}$ions in $\mathrm{HZr}_{2}\left(\mathrm{PO}_{4}\right)_{3}$, it is assumed that the $\mathrm{H}$-bonding interaction between the zirconium phosphate surface and the hydroxyl groups of alcohol molecules plays an important role in the sensing process; namely, there would be interactions between electron donors and acceptors. Moreover, when the sensitivity of ketones and aldehydes was measured (Figs. 7(e)-7(i)), it was clear that their sensitivities were extremely low, as it is considered that there is a difference in H-bonding interaction between alcohol and ketones or aldehydes. That is, SPR with alkyl thiol-zirconiumphosphate hybrid layers could be used for the selective detection of alcohols. On the other hand, there are a number of studies on $\mathrm{TiO}_{2}$ surfaces. ${ }^{(14)}$ The zero point charge of $\mathrm{TiO}_{2}$ is 6.7.(15) The relative dielectric constant of $\mathrm{TiO}_{2}(85.8$ at $298 \mathrm{~K})$ is close to that of water $(80.1$ at $293 \mathrm{~K}){ }^{(16)}$ Therefore, the surface of $\mathrm{TiO}_{2}$ has a strong affinity to the $\mathrm{OH}$ group of alcohol. Thus, inorganic layer optimization can provide further possibilities for developing new specific gas-sensing devices. Further studies to investigate these issues and the selectivity are in progress.

\section{Conclusions}

The major observations in our study of the SPR sensor with thiol-zirconium phosphate and thiol- $\mathrm{TiO}_{2}$ hybrid layer are as follows: (a) the peak of the incident angle for SPR spectra changed after gas adsorption, and the magnitude of the shift was dependent on gas concentration and the type of adsorbates; (b) for SPR sensor with a $\mathrm{TiO}_{2}$ hybrid, the lower limit of detection was approximately $4 \mathrm{ppm}$; (c) the sequence of the sensitivity was as follows: 2-butanol > 1-propanol > ethanol > methanol; and (d) for the SPR sensor with zirconium phosphate, the sensitivity of aldehydes and ketones was extremely low and selective detection of alcohols could be achieved. 


\section{References}

1 H. Raether: Physics of Thin Films, Vol. 9 (Academic Press, New York, 1977) p. 145.

2 H. Raether: Springer Tacts in Modern Physics, Vol. 111, ed. G.Hohler (Springer-Verlag, Berlin, 1988) p. 4.

3 I. Pockrand, J. D. Swalen, S. Santo, A. Brillante and M. R. Philpott: J. Chem. Phys. 69 (1978) 4001.

4 Y. J. Chen and G. M. Carter: Appl. Phys. Lett. 41 (1982) 307.

5 C. Nylander and I. Lundstrom: Sens. Actuators 3 (1982/1983) 79.

6 S. Miwa and T. Arakawa: Thin Solid Films 281/282 (1996) 466.

7 M. G. Manera, G. Leo, M. L. Curri, P. D. Cozzoli, R. Rella, P. Siciliano, A. Agostiano and L. Vasanelli: Sens. Actuators, B 100 (2004) 75.

8 G. A. Sotzing, J. N. Phend, R. H. Grubbs and N. S. Lewis: Chem. Mater. 12 (2000) 593.

9 N. Guernion, B. P. J. de Lacy Costello and N. M. Ratcliffe: Synth. Met. 128 (2002) 139.

10 T. Arakawa, A. Kawabayashi and T. Saga: Sens. Actuators, B 108 (2005) 899.

11 K. Ideta and T. Arakawa: Sens. Actuators, B 13/14 (1993) 384.

12 T. Ishihara and T. Arakawa: Sens. Actuators, B 91 (2003) 262.

13 J. B. Goodenough, H. Y.-P. Hong and J. A. Kafalas: Mater. Res. Bull. 11 (1976) 203.

14 T. W. Healy and D. W. Fuerstenan: J. Colloid Sci. 20 (1965) 376.

15 G. D. Parfitl: Prog. Surf. Membrane Sci. 11 (1976) 181.

16 H. P. Bohem: Discuss. Faraday Soc. 52 (1971) 264. 